of treatment in a $70 \mathrm{~kg}$ individual **Patients underwent EBV screening as part of generic pre-screening bloods.

\begin{tabular}{|c|c|c|c|c|}
\hline $\begin{array}{l}\text { No of } \\
\text { patients } \\
\text { screened }(n)\end{array}$ & $\begin{array}{l}\text { Initial } \\
\text { treatment } \\
\text { agent } \\
\text { considered }\end{array}$ & $\begin{array}{l}\text { No of patients in } \\
\text { whom treatment was } \\
\text { altered (n) }\end{array}$ & $\begin{array}{l}\text { Agent } \\
\text { changed } \\
\text { to }\end{array}$ & $\begin{array}{l}\text { Average increased } \\
\text { cost expenditure per } \\
\text { patient }\end{array}$ \\
\hline 22 & Thiopurine & 12 & Biologic & f9942* \\
\hline 6 & Biologic & - & - & - \\
\hline 5 & $-* *$ & 1 & Biologic & f4112* \\
\hline 1 & $\begin{array}{l}\text { Combination } \\
\text { therapy }\end{array}$ & - & - & - \\
\hline
\end{tabular}

Of the 13 individuals who had their therapy switched 8 (62\%) were male and 10 (77\%) were Caucasian.

Conclusions Overall EBV naïve status resulted in initiation of therapy different to the initial agent considered in $38 \%$ of patients, producing an overall cost expenditure of approximately $£ 123416$ with treatment more likely to be switched in male and Caucasian patients.

However, the absolute risk of lymphoma remains low and given the cost, the average age of patients being associated with poorer prognosis and increased likelihood of subsequent antibody development to biologic therapy in patients without initial thiopurine treatment, is the practise of EBV screening in IBD management clinically relevant?

\section{PWE-002 EVALUATING THE ROLE OF MR ENTEROGRAPHY IN THE MANAGEMENT OF PATIENTS WITH CROHNS DISEASE}

Wafaa Ahmed*, Bhavin Kawa, Gautom Baruah, Sukanya Ghosh, Eleanor Warner, Bijay Baburajan. Maidstone And Tunbridge Wells NHS Trust, Kent, UK

\subsection{6/gutjnl-2018-BSGAbstracts.134}

Introduction Magnetic resonance enterography (MRE) is widely available and is being increasingly utilised in the management of patients with Crohns disease (CD). Free of ionising radiation, it is particularly valuable in sequential imaging. In addition, it is an attractive, non-invasive option in the investigation of patients suspected to have small bowel CD. We sought to investigate whether MRE in patients with CD alters patient management as well as its utility in the diagnosis of CD.

Methods PACS was interrogated to identify patients undergoing MRE across two sites in a trust from March to April 2017. Unique patient IDs were cross referenced with Telepath, Allscripts and Endobase databases for demographic data, results of faecal calprotectin (FC) and clinical outcomes. MR protocol was standardised and calprotectin measurement has been available since 2016 .

Treatment change was defined as treatment initiation, escalation or cessation and referral for surgery. Patients being worked up for CD were those that had diarrhoea, abdominal pain or weight loss as their primary indication for MRE.

Results There were 111 MRE carried out over the 3 month period, mean age 38.2 (12-82), male 44 (39.6\%), female 67 (60.4\%).Of those $57(51.3 \%)$ had established CD and 53
(47.7\%) were for investigation of suspected CD. In this group, the results of the MRE influenced the management of 39 cases $(68.4 \%)$ vs $18(31.6 \%)$ with no change. $30(52.6 \%)$ patients had a new treatment or treatment escalation, 6 $(10.5 \%)$ had their treatment stopped and 3 (5.3\%) were referred for surgery. 24 patients with $\mathrm{CD}$ had FC done within 2 months of MRE. Interestingly all of those with a FC $<250 \mathrm{ug} / \mathrm{g}$ had a normal MRE $(\mathrm{n}=8)$.

In patients being worked up for CD, 42 (79.2\%) of the MREs were normal and $2(3.7 \%)$ were highly suspicious for $\mathrm{CD}$, the rest showed non-specific inflammation and other findings including liver haemangioma, cholecystitis, jejunal diverticulitis and a carcinoid tumour. Surprisingly, MRE was used as the initial investigation in $9(16.9 \%)$ patients, in which there was one case of cholecytitis but the rest were negative. As in patients with CD, no patient with a FC $<250 \mathrm{ug} / \mathrm{g}$ had a positive MRE.

Conclusion MRE results influence treatment decisions in CD, facilitating changes in management including withdrawal of biologics. It is seemingly ineffective as a diagnostic test for small bowel $\mathrm{CD}$ and calprotectin may be more useful in that context. This study is limited by the exclusion of patients with Crohn's disease who have not had MRE but these findings suggest potential cost savings if MRE is reserved for patients with CD suggested by other means such as faecal biomarkers or endoscopy.

\section{PWE-003 ACETARSOL IS AN EFFECTIVE OPTION IN THE MANAGEMENT OF REFRACTORY PROCTITIS}

${ }^{1}$ Konstantinos Argyriou*, 'Richard Ingram, ${ }^{2}$ Rachel Blatt, ${ }^{2}$ Patrick Wilson, 'Sunil Samuel, ${ }^{1}$ Christopher Hawkey, ${ }^{1,3}$ Gordon Moran. ${ }^{1}$ Nottingham Digestive Diseases Center, Queen's Medical Centre Campus, Nottingham University Hospitals NHS Trust, Nottingham, UK; ${ }^{2}$ Pharmacy Department, Queens Medical Center Campus, Nottingham University Hospitals NHS Trust, Nottingham, UK; ${ }^{3}$ National Institute of Health Research Biomedical Research Centre in Gastrointestinal and Liver Diseases at Nottingham University Hospitals and the University of Nottingham, Nottingham, UK

\subsection{6/gutjnl-2018-BSGAbstracts. 135}

Introduction There is an urgent need for superior topical therapy for mesalazine-refractory ulcerativeproctitis. Two small preliminary cohort studies have suggested good clinical efficacy data for organic arsenic derivatives such as acetarsol in refractory ulcerative proctitis. Our aim was to describe the effectiveness and tolerability of acetarsol suppositories in a cohort of inflammatory bowel disease (IBD) patients.

Methods We performed a retrospective cohort study by prospectively reviewing maintained clinical records for all patients exposed to acetarsol at the IBD clinic in Nottingham University Hospitals from 2004 to 2017. Response was determined as clinical improvement or improvement in endoscopic appearance. Serum arsenic and C-reactive protein levels were reviewed when available. Non parametric statistical analysis was performed. Data are presented as median and interquartile range Results 35 patients were prescribed acetarsol suppositories (28 with proctitis, 5 with left-sided colitis, 1 with diversion colitis and 1 with chronic pouchitis). Twenty were males with median age of 44 (34) years and disease duration of 7 (19) years. Nearly all patients had failed mesalazine or corticosteroid-based topical therapy with $20(57.1 \%)$ being refractory to immunotherapy and $3(8.6 \%)$ to anti-TNF therapy. Acetarsol $250 \mathrm{mg}$ bd for at least 4 weeks was prescribed in $75 \%$ of the cases. Median treatment duration was 56 days (28). Sixteen 
patients were exposed to acetarsol more than once. $76.7 \%$ of patients achieved clinical response. $3 / 35$ patients had an endoscopic assessment with two of three patients showing endoscopic improvement. $33.3 \%$ patients required treatment escalation following acetarsol exposure with two undergoing subtotal colectomy. Five patients (14.3\%) stopped acetarsol due to side effects. One patient experienced vomiting, palpitations and sweating, and the other four experienced headache, vomiting, anal itching and paresthesia. Median serum arsenic level was $728.25(872) \mathrm{nmol} / \mathrm{l}(<130 \mathrm{nmol} / \mathrm{L})$. Serum arsenic levels were not correlated with patient clinical response nor the need for treatment escalation.

Conclusions Acetarsol suppositories could be an effective and tolerable option in the management of refractory proctitis. A definitive study is urgently warranted to thoroughly investigate the clinical efficacy and safety of this promising drug.

\section{PWE-004 TAPERING WITH BUDESONIDE - LABEL RECOMMENDATION VERSUS CLINICAL REALITY}

${ }^{1}$ Philip Becker*, ${ }^{1}$ Robert Hofmann, ${ }^{2}$ Benny Chemnitz-Nielsen. ${ }^{1}$ Tillotts Pharma; Medical Affairs, Rheinfelden, Switzerland; ${ }^{2}$ Kantar Sifo, Göteborg, Sweden

\subsection{6/gutjnl-2018-BSGAbstracts. 136}

Introduction Symptoms of steroid withdrawal may be avoided in most patients by tapering the dose over time when deciding to discontinue treatment. Patients exposed to steroid therapy, even if only for a short period of time are very likely to have diminished hypothalamic-pituitary-adrenal (HPA) axis function. Despite primarily local action, budesonide can also affect the HPA axis. Tapering of budesonide allows the adrenal glands time to return to their normal patterns of secretion. This is a precaution and highlighted in the Entocort* Summary of Product Characteristics recommendation where it is stated 'when treatment is to be discontinued, the dose should normally be reduced for the last 2 to 4 weeks of therapy'. The presented study was investigating tapering habits for budesonide prescriptions in Crohn's disease (CD) among gastroenterologists in Europe.

Methods An online survey** was conducted among a total sample of 161 gastroenterologists in 8 countries. The data collection took place from November to December 2016. The physicians in the sample received a link to an online questionnaire (local language) on a secure platform via email. The questionnaire on prescribing practises included the following questions with regards to the use of budesonide for the treatment of CD: (i) Do you normally recommend tapering dose in the last 2-4 weeks of budesonide treatment? (ii) Do you recommend tapering over 2, 3, or 4 weeks? (iii) Do you recommend tapering to either $6 \mathrm{mg}$ then $3 \mathrm{mg}$, straight to $3 \mathrm{mg}$ or to $6 \mathrm{mg}$ only?

Results Of the 161 gastroenterologists surveyed, the majority (81\%) tapered when prescribing budesonide; the countries where the response rate was $\geq 80 \%$ included Finland (100\%), Norway (88\%), Czech Republic (82\%), Denmark (82\%) and Spain (80\%). Most gastroenterologists tapered over a 4 week time period $(78 \%)$, with a small minority over other time schedules (2 or 3 weeks). The most commonly used tapering schedule was 9-6-3-0 mg (91\%), with only 5\% and $4 \%$ of gastroenterologists tapering using a $9-6-0 \mathrm{mg}$ and $9-3-0 \mathrm{mg}$ schedule, respectively.
Conclusions The majority of gastroenterologists surveyed are adhering to the budesonide label recommendation of tapering over a 2-4 week period when prescribing budesonide. The reasons behind $20 \%$ of prescribers deviating from the product labels needs further research to be addressed.

* The rights to Entocort, including the rights to the trademark, are owned by Tillotts Pharma AG except for the USA.

*Financial sponsorship was provided by Tillotts Pharma AG.

\section{PWE-005 VEDOLIZUMAB DOSE ESCALATION AS A WAY OF RECAPTURING RESPONSE IN PATIENTS WITH INFLAMMATORY BOWEL DISEASE}

'S Birdi ${ }^{*},{ }^{1,2} \mathrm{M}$ Sierra Morales, ${ }^{2} \mathrm{MA}$ Samaan, ${ }^{1} \mathrm{C}$ Brown-Clarke, ${ }^{1} \mathrm{~A}$ Stanton, 'S Ray, 'I Koumoutsos, 'J Mawdsley, 'S Anderson, 'Sandersdon, 'PM Irving. 'Guy's and St Thomas' Hospital NHS Trust,Department of Gastroenterology, London, UK; ${ }^{2}$ Hospital Universitario Ramón y Cajal, Madrid, Spain

\subsection{6/gutjnl-2018-BSGAbstracts. 137}

Introduction Increasing vedolizumab (VDZ) dosing frequency to recapture response has been shown to be effective in clinical trials but there is limited real-life data from the clinical practice. In this study we assessed whether VDZ dose escalation helped recapture response in a large cohort of patients in a tertiary referral IBD centre.

Methods A retrospective cohort study was performed by reviewing prospectively recorded clinical data for patients who received VDZ between November 2014 and October 2017. Patients who had sub-optimal response and had been escalated to 6 or 4 weekly infusions were identified. Data collected for demographics, previous biologic exposure, concomitant immunomodulators (IM), steroid use (SU), clinical disease activity for CD (HBI) and UC (SCCAI), and CRP levels at baseline, 12 and 24 weeks after dose escalation.

Of the total 139 patients on VDZ, $36(27 \%)$ had been escalated to Q4 (30) or Q6 (6), of whom 5 were further escalated to Q4 (72\% male, median age 44 , previous biologics exposure $81 \%$, 49\% concomitant IM and $16 \%$ SU at time of escalation). 18 patients had CD (50\%), 14 UC (39\%), and 4 (11\%) IBD-U which were included in the UC group for the purpose of analysis.

Duration of VDZ before and after dose escalation with a median of $7 \mathrm{~m}$ (ranges 0-22, 2-25 respectively). Currently $76 \%$ remain on VDZ after dose escalation (median $7 \mathrm{~m}$ after escalation).

Clinical response was defined as HBI or SCCAI reduction $>3$. Remission as $\mathrm{HBI}<5$ or SCCAI $<3$. Paired HBI, SCCAI, CRP values at baseline, week 12 and 24 were compared using Wilcoxon signed-rank test

Results Patients with CD had a median HBI of 4 (range 027), 4 (0-29) and $3(0-8)$, at baseline, 12 and 24 weeks.

In UC group, the median SCCAI was 6 (range 0-11); 4.5 (1-11), and 4 (0-10), at baseline, 12 and 24 weeks.

CRP for both groups at baseline was a median of 6 (123), $5(1-46)$ at w12, and $2(1-17)$ at w24.

HBI and SCCAI at baseline, 12 and 24 weeks after dose escalation

Statistically significant differences were noted in the UC group between SCCAI at baseline and after 24 weeks ( $p$ 0.01) and overall CRP at baseline and $24 \mathrm{w}$ (p 0.04). 